Meta

Journal des traducteurs

Translators' Journal

\title{
Traduction et création
}

\section{Christine Bagge}

Volume 29, numéro 2, juin 1984

URI : https://id.erudit.org/iderudit/001944ar

DOI : https://doi.org/10.7202/001944ar

Aller au sommaire du numéro

Éditeur(s)

Les Presses de l'Université de Montréal

ISSN

0026-0452 (imprimé)

1492-1421 (numérique)

Découvrir la revue

Citer cet article

Bagge, C. (1984). Traduction et création. Meta, 29(2), 135-142.

https://doi.org/10.7202/001944ar

Ce document est protégé par la loi sur le droit d'auteur. L’utilisation des services d'Érudit (y compris la reproduction) est assujettie à sa politique d'utilisation que vous pouvez consulter en ligne.

https://apropos.erudit.org/fr/usagers/politique-dutilisation/
Cet article est diffusé et préservé par Érudit.

Érudit est un consortium interuniversitaire sans but lucratif composé de l’Université de Montréal, l'Université Laval et l'Université du Québec à Montréal. Il a pour mission la promotion et la valorisation de la recherche. https://www.erudit.org/fr/ 


\section{TRADUCTION ET CRÉATION}

Christine BagGe

Comme le fait remarquer Susan Bassnett-McGuire (1980), la traduction était jusqu'à il n'y a pas très longtemps considérée comme " the rendering of a source language text into the target language so as to ensure that the surface meaning of the two will be approximately similar... ». Les carences de cette définition, cependant, sont immédiatement évidentes à tout spécialiste du domaine. Entre autres, cette définition ne tient aucun compte des qualités créatrices de cette activité. Birago Diop, dans les Contes d'Amadou Koumba, offre la preuve indubitable que la traduction est aussi création.

Lors de la rédaction de ses Contes d'Amadou Koumba, Birago Diop a dû faire face à deux difficultés essentielles : d'une part le problème, que doit affronter tout traducteur, de l'impossibilité de rendre dans une langue européenne une histoire traditionnelle sans perdre beaucoup de la richesse d'expression de la langue vernaculaire (Diop 1967); d'autre part le passage d'une forme à une autre, la transcription sur papier d'une tradition entièrement orale, qui de nature "depended on qualities such as tone of voice, emotional expression, gestures, mime, repetition, interpolated song and dance " (Diop 1967). Il s'agit donc là pour Birago Diop, non pas tout simplement de transcrire dans une autre langue le contenu des contes, ce que nombre de linguistes et d'anthropologues ont fait, mais de "traduire" ces contes, de rendre accessible à une autre civilisation une forme d'art didactique africain tout à fait spécifique et unique en son genre. C'est de par cet aspect de son oeuvre et malgré ses protestations modestes — - Si je n'ai pu mettre dans ce que je rapporte l'ambiance où baignait l'auditeur que je fus... " (Diop 1969) - que ce " tisserand malhabile " (BD : 12) s'élève au rang de l'artiste créateur. Les Contes d'Amadou Koumba donnent au lecteur une impression artistique qu'il est difficile d'expliquer. Il est tout de même intéressant, pour essayer de l'analyser, de comparer les contes que Birago Diop dit d'Amadou Koumba avec "Sarzan " né uniquement de l'imagination de l'auteur, de les comparer également avec les traductions de contes traditionnels africains de Blaise Cendrars et avec des contes folkloriques transcrits en anglais par des Africains et par des Anglais.

Cette étude évidemment laissera de côté le fond, puisque nul ne peut en nier l'authenticité. Par là s'entend que même au cas où tous ces contes n'auraient pas été racontés à Birago Diop par Amadou Koumba (il serait bien difficile de le prouver !), ils font sans aucun doute partie de l'héritage que la tradition africaine a légué de bouche à oreille à la postérité. Même l'intrigue de "Sarzan ", fondée sur une situation réelle et contemporaine - le retour du Noir africain "civilisé » dans son pays natal — n'est pas le siège de l'originalité de Diop. C'est dans la structure de ses contes, la caractérisation des personnages et le style, en un mot dans la forme, que Birago Diop se montre " créateur".

Comme l'explique J.A. Hutchinson (JAH : 14) il y avait des rites traditionnels que le griot devait observer en racontant ses histoires, telles que la formule d'introduction destinée à créer l'atmosphère propice au surnaturel. Celle-là prenait quelquefois la forme d'une invocation, introduisant ainsi un élément presque religieux dans le conte ; 
de même des rites finals terminaient le conte. Cette absence de la grande introduction et de la conclusion traditionnelles peut être reprochée à Diop comme étant une concession qu'il fait au goût occidental qui ne s'accommoderait peut-être pas très bien de ce genre de prose. Pourtant le grief ne devrait pas être trop sévère puisque aucun des autres transcripteurs de contes, que ce soit d'expression française ou anglaise, ne respecte cette coutume.

En fait, ces derniers ne se limitent qu'au strict minimum et ne font très souvent qu'un simple récit des actions. Une illustration parmi tant d'autres pourrait être le début de ce conte transcrit par Cendrars : "Un jeune homme avait du miel. Il le donne à sa grand'mère. Celle-ci le mange. À son retour, il le réclame. Elle l'avait mangé. Elle dut lui donner du grain. Il l'emporte. Des poulets viennent " (Cendrars 1963). Diop par contre, dans son introduction souvent détaillée, ou dans ses digressions plus ou moins longues, plante un décor, donne des indications scéniques ou encore permet une appréhension immédiate du ton du conte. Ainsi, par exemple, l'introduction de l'héritage décrit parfaitement le cadre où la scène se déroule, de même qu'elle traduit cette impression de calme et de repos que l'on éprouve dans un tel lieu : "La douce journée qui s'achevait était l'image de la vie du vieux Samba : calme et limpide, remplie de labeur, de sagesse, de bonnes actions. Les souffles qui portaient la nuit s'attardaient au faite du tamarinier » (BD : 163). Il n'y a pas de doute quant à l'origine de ce trait caractéristique de la structure des Contes d'Amadou Koumba. Il est de Diop et uniquement de lui puisque cet artifice ne se trouve pas dans les contes transcrits par d'autres auteurs, africains ou non, mais que par contre il est présent dans "Sarzan", ce fruit de l'imagination de Diop : " Les ruines s'amoncelaient indistinctes des termitières, et seule une coquille d'oeuf d'autruche fêlée et jaunie aux intempéries indiquait encore, à la pointe d'un haut piquet, l'emplacement du mirab de la mosquée qu'avaient bâtie les guerriers d'El Hadj Omar » (BD : 173). Cette description encore une fois situe le conte et en même temps lui donne dès le départ son ton et sa couleur.

En ce qui concerne les indications scéniques, la comparaison entre la transcription d'un même conte, "Vérité et mensonge ", par Cendrars et par Diop met l'accent sur le talent littéraire de ce dernier. Cendrars écrit : «Tant et si bien que tous tes aïeux sont là et que je ne sais plus qui entendre? Mais pour ne pas exagérer ton embarras, dis moi seulement qui, de ton père ou de ta femme, je dois ressusciter?" (BC: 316$)$. Birago Diop, par contre, insère au milieu de ce discours une petite digression qui permet de voir totalement la scène, de connaître la réaction du roi sous le flot des paroles du Mensonge. "Tant et si bien que tes ancêtres et les aïeux de leurs ancêtres sont maintenant à la sortie de la tombe de ta femme. Bour-le-Roi regarda ses conseillers, et les notables regardèrent le roi. L'étranger avait bien raison de dire que les choses se gâtaient. Bour regarda Fènele-Mensonge, et les vieux le regardèrent. Que fallait-il faire ? Pour te tirer d'embarras, pour t'éviter d'avoir trop à choisir, dit Fène-le-Mensonge, indique-moi qui, de ta femme ou de ton père, tu veux que je fasse revenir " (BD : 134).

Birago Diop se sert aussi très souvent de ses longs préambules comme d'un habile artifice pour raconter deux ou trois contes dans un seul. Ainsi par exemple dans « Mauvaise compagnie IV ", la première mésaventure du crapaud est arrivée à l'ancêtre de celui qui est le protagoniste de la deuxième péripétie. À peu près le même principe est appliqué dans "N'Gor-Niébé » ou "La biche et les deux chasseurs " pour ne citer que ceux-là. Cet artifice nous épargne la monotonie qui naît inévitablement de la longue succession des aventures qui arrivent à un même personnage, comme dans "The Hare" (Feldman 1970) où H.A. Junod nous retrace en vingt pages toute une série d'épisodes dont le lièvre est protagoniste. Contrairement à Birago Diop, il ne cherche pas du tout à lier d'aucune façon les différentes parties l'une à l'autre. Les transitions se réduisent à 
une phrase simple telle que : "The wily trickster Hare took the hoe and then went to look for Grey Antelope " (SF : 146). Une longue liste de ces phrases transitions montrerait à quel point elles sont brèves et sans effet spécial.

D'autre part, Birago Diop ne se contente pas, comme les autres traducteurs en français ou en anglais, de l'évidence de l'action; il en donne souvent le mobile. Cette explication, qu'elle soit à tendance philosophique comme dans cet exemple où nous suivons le processus mental de Fari l'ânesse : "Mais comment disposer sans risques de toutes ces bonnes choses qui appartenaient aux hommes? Un seul moyen peut-être : se faire homme soi-même. Mais l'homme cède-t-il volontiers à son semblable ce qui lui appartient, ce qu'il a obtenu à la sueur de ses bras ? À la femme peut-être ... Fari décida donc de ... se métamorphoser en femme, sa suite également " (BD : 15), ou plutôt cocasse comme l'annonce du retour de Narr : "Les choses auraient pu peut-être durer toujours ainsi si Narr avait péri en chemin ; s'il avait été pris là-bas vers l'est dans un royaume bambara, peulh ou haoussa et maintenu en esclavage ; ou s'il avait préféré demeurer, le restant de ses jours, près de la Kaaba pour être plus près du paradis. Mais Narr revint au beau jour... » (BD : 17), cette explication donc, ne manque jamais d'humour.

Par ailleurs, Birago Diop fait aussi un grand usage de la digression en nous donnant des remplissages pittoresques : "Force fut au taciturne solitaire, dont l'humeur était moins changeante que la couleur de la peau, de répondre à la politesse. Car ... l'on peut payer cette dette sans s'appauvrir. Et puis, rendre un salut n'a jamais écorché la bouche" (BD : 60). Ces remplissages prennent également la forme de commentaires personnels à première vue superflus : "Lorsqu'on est bien élevé, répond-on que l'on va mal à quelqu'un qui s'inquiète de votre santé ? Cela ne s'est jamais entendu et ne s'entendra jamais tant qu'il y aura des gens qui ont reçu un semblant d'éducation " (BD : 68). Ces digressions-commentaires on ne les trouve nulle part dans les transcriptions habituelles. Pour prouver la réalité de cette constatation il n'est que de comparer encore une fois deux passages de "Vérité et mensonge ". Alors que Cendrars se contente de décrire en une phrase pas très longue : " Alors, la femme de l'hôte, violemment irritée contre ces étrangers qui se permettaient de se mêler des affaires de son ménage, les jeta finalernent à la porte " (BC : 315), Birago Diop s'arroge tout un paragraphe teinté d'humour : «Alors la femme se mit dans une colère folle, et, menaçant d'ameuter tout le village, intima à son mari l'ordre de mettre à la porte ces étrangers impertinents qui s'occupaient de son ménage et se mêlaient de donner des conseils, sans quoi elle s'en retournerait sur l'heure chez ses parents " (BD : 131). À partir d'un mot seulement, quelquefois, Birago Diop brode et utilise ainsi au maximum toute la substance de ce mot. Ainsi pour expliquer qu'il est difficile de confier de la viande à une hyène sans qu'elle la mange, écrit-il : "C'était là chose plus difficile que de faire garder un secret à Narr-leMaure aux oreilles rouges. C'était là chose plus difficile que de confier une calebasse de miel à un enfant sans qu'il y trempe au moins le petit doigt. Autant essayer d'empêcher le soleil de sortir... " (BD : 94). Un Africain d'expression anglaise, par contre, se contente de : "...the young man perceived that it would not become possible for him to have the wine " (Hughes 1969). Bien que Birago Diop ne dispose pas des mêmes moyens que les conteurs traditionnels - gestes, modulations de la voix, mimiques - il ne renonce pas. Par la structure même de ses contes, il arrive à leur rendre un peu de ces qualités qui en faisaient un véritable spectacle divertissant en même temps qu'instructif. Cela, il le fait quelquefois en reprenant à son compte des moyens traditionnels comme par exemple l'emploi du refrain (BD : 54, 121, 150) mais aussi et surtout par les usages multiples qu'il fait de la digression. Par les vertus théâtrales, divertissantes, explicatives et commentatrices de ces digressions, Diop conserve, autant que possible, à la structure des contes leurs qualités originelles. Il crée un nouveau mode d'expression pour évoquer 
une atmosphère traditionnelle. Mais ce n'est pas seulement à travers la structure de ses contes que Birago Diop parvient à ce prodige. Sa façon de peindre et de donner vie à ses personnages y contribue grandement.

Alors que dans la plupart des transcriptions de contes traditionnels, les personnages sont plats, stéréotypés et presque symboliques, Diop parvient à leur conférer une existence réelle. Ses personnages se classent en trois catégories : les humains, les animaux et les abstractions, comme dans tous les contes traditionnels. Cependant, ces personnages ne sont que nommés ou peut-être qualifiés par un adjectif ou deux dans la plupart des transcriptions, par exemple, "The Wily Trickster Hare" (SF : 144); ou bien quelques rares détails sont donnés sur leur apparence : "...sa favorite se nommait Morongoé. Boulané avait sur la poitrine l'image d'une lune en son plein ; c'est pourquoi on l'avait surnommé Khoédi-Séboufeng (Lune sur la poitrine) » (BC: 323), ou sur leur identité : "Samba Guélâdio Diêgui était fils de Tuélâdio, roi du Fouta " (BC : 335). D'une façon générale, donc, les personnages ne sont caractérisés que par leurs actions et sont toujours vus de l'extérieur. Birago Diop, par contre, leur donne une dimension supplémentaire en utilisant soit une description réaliste et détaillée comme par exemple la peinture sensuelle qu'il fait de la femme africaine dont l'importance du rôle dans ces sociétés est bien connu : "Ses seins pointaient sous sa camisole brodée, sa croupe rebondie tendait son pagne de n'galam. À chacun de ses gracieux mouvements, tintaient ses ceintures de perles et son parfum entêtant agaçait les narines de Demba" (BD : 22). Cette dimension il la leur donne aussi en les présentant quelquefois de l'intérieur et non plus de l'extérieur. Il est à ce propos intéressant de comparer la description des réflexions de Fari l'ânesse, déjà citée (BD : 15), quand elle décide de ce qu'elle doit faire pour sauver sa race de la faim, avec ceci : "What could the hungry frogs do ? They were at the mercy of this most rapacious and ferocious animal " (LH : 82). Et l'histoire continue racontant ce qu'elles ont fait sans jamais expliquer comment l'idée leur en est venue. De même, alors que dans "Vérité et mensonge "Cendrars passe toute de suite à l'action, Birago Diop prend tout un paragraphe pour expliquer ce qui suscite la première remarque de Mensonge (BD : 129).

Birago Diop, en outre, donne très souvent un détail cocasse et inattendu qui campe le personnage et qui le rend inoubliable. Ceci se produit entre autres lorsqu'il parle des yeux du crabe que ce dernier " rabattit puis releva " $(B D: 69)$ ou encore de la sauterelle "aux coudes pointus" (BD : 74). Même dans un conte moins élaboré que les autres comme "Le salaire", on lit que le lièvre "Leuk se frotta le menton, se gratta l'oreille... " (BD : 103). Incidemment, on peut remarquer que ces détails imagés concernent surtout les animaux et il est un fait que ce sont les animaux plus que les humains qui donnent aux histoires leur couleur et leur vie (voir AH : 25).

Enfin, Birago Diop donne des noms à ses personnages. Alors que les deux abstractions personnifiées ne sont pour Cendrars que "Le Mensonge " et "La Vérité ». Par moments, même, ce n'est plus que par leurs prénoms que Diop les nomme : "Fène et Deug continuèrent donc leur voyage » (BD : 131), «En route, Fène dit à Deug " (BD : 132), "Fène fit bâtir une case... " (BD : 133). Cette différence est déjà remarquable dans le titre du conte lui-même. Tandis que pour Cendrars, il s'agit de "Le Mensonge et la vérité ", c'est-à-dire de deux abstractions, bien que personnifiées, pour Diop, ce sont d'emblée " Mensonge et vérité ", c'est-à-dire Fène et Deug. Par cette suppression de l'article, Diop les incarne immédiatement. Diop d'ailleurs personnifie les abstractions même quand elles ont un rôle minime dans l'action : "Quand l'homme dit à son caractère : "Attends-moi ici ", à peine a-t-il le dos tourné que le caractère marche sur ses talons" (BD : 16). "Mais au milieu de la nuit, son secret, qui s'était mis en travers du 
couscous et du mouton dont il s'était gavé, l'étouffait " (BD : 17). "Cette compagne c'est l'Envie à la voix aigre et acide du jus de tamarin " (BD : 33).

En outre, Birago Diop s'arrange toujours pour garder les deux protagonistes sur lat scène. Ce phénomène est rendu apparent par une simple comparaison entre ce petit épisode raconté par Cendrars : "Qui pensez-vous donc qui commande ici ? Apparemment, dit la Vérité, ce sont les enfants » $(B C: 315)$ et le même épisode vu par Birago Diop : "Qui pensez-vous donc qui commande dans ce village... ? Prudemment, Fène-leMensonge garda le silence et n'ouvrit pas la bouche ; et Deug-la-Vérité fut bien obligée comme convenu, de donner son avis... " (BD : 131). Bien que le Mensonge ne dise rien, il est toujours présent.

Finalement, un aspect de la caractérisation de ces personnages qu'il ne faut pas négliger, ce sont les dialogues. Les dialogues étant très réalistes, les personnages gardent toute leur authenticité. La preuve la plus convaincante de ce fait est sans aucun doute une comparaison entre les dialogues de Cendrars et ceux de Diop dans "Vérité et mensonge ". Il serait évidemment beaucoup trop long de citer tous les exemples, mais un ou deux pris au hasard suffiront. Ainsi lorsque le mari dit dans le conte de Cendrars : "Pensez-vous que ce soit là le fait d'une bonne ménagère ? » $(\mathrm{BC}: 315)$ il ne laisse rien percer de sa colère et de son indignation alors que ces deux sentiments lui sont rendus sous la plume de Diop par la répétition saccadée de la même phrase : "Est-ce là une bonne épouse? Est-ce là le fait d'une femme généreuse ? Est-ce là une bonne ménagère? " (BD : 130). Ou bien encore, alors que Cendrars se contente de constater : " $\grave{A}$ ces mots, le chef se mit dans une terrible fureur et fit chasser ces étrangers, si impertinents " (BC: 315), Diop fait ressortir la colère du vieillard à travers ses paroles mêmes : «Vous êtes insolents, dit le vieillard en courroux. Sortez de ce village, partez, partez tout de suite, sans quoi vous n'en sortirez plus. Allez-vous-en, allez-vous-en" (BD : 131-132).

En fin de compte, si Birago Diop respecte la tradition en ne donnant pas trop de profondeur psychologique à ses personnages, il ne se limite pas à cela. Par ses descriptions réalistes et détaillées, par l'exploration superficielle de l'intellect de ses protagonistes, en les personnifiant par des noms et en les affublant d'un langage expressif, Birago Diop arrive à exprimer par écrit ce que les griots faisaient par gestes ou intonation de la voix : il crée des personnages tangibles qui existent autrement que par leurs actions. Mais pour rendre pleinement compte de leur contexte initial, Birago Diop a aussi recours à son style.

À ce sujet, Diop est très critiqué parce que pour donner une vie à un aspect de la civilisation d'Afrique noire il emploie l'outil occidental s'il en est un : le langage de l'Ouest. Mais encore une fois, il n'est pas le seul et déterminer s'il a tort ou raison est une question toute subjective qui dépasse le cadre de cette étude.

Du point de vue de son style, l'avantage de Diop est d'être africain. Ainsi par exemple, son imagerie est quelquefois traditionnelle : "...il s'était aigri comme du lait qu'un génie a enjambé... » (BD : 34) « Leuk-le-lièvre, le malin et malicieux lièvre, dont la conscience est aussi mobile que les deux savates qu'il porte accrochées à la tête, du jour où il les enleva pour mieux courir, et qui, depuis, lui servent d'oreilles... " (BD : 50-51) "la cervelle de poulet trouble l'intelligence, comme une motte d'argile fait d'une calebasse d'eau "(BD : 75). Cette imagerie est aussi très souvent animiste. Dans les contes, comme Senghor l'explique, " tout a une vie ". C'est ainsi que Diop parle de la "lune fatiguée qui confiait à Bouki-l'Hyène la garde des troupeaux " $(\mathrm{BD}: 65)$ et enfin pour ne pas rendre trop longue une énumération qui n'est en rien limitative, il suffira de donner une autre illustration : " le soleil, traînant tout le long du jour dans ses champs, ne paraissait plus pressé de regagner sa demeure » (BD : 39). Cependant, si au départ ce n'est que l'esprit animiste qui inspire Diop, il sait s'en emparer et l'exploiter au maximum jusqu'à lui 
donner une touche toute personnelle, comme dans ce poétique petit passage : "Sous le poids de la rosée, l'herbe courbait encore la tête. De jeunes ruisseaux déjà réveillés se disputaient et jouaient à cache-cache. Le soleil, faisant son ménage, balaya de ses rayons la rosée... " (BD : 167). Birago Diop donc ne se contente pas de traduire littéralement des images africaines, comme l'exemple suivant le prouve : "...de passer sa colère sur Koumba sa femme, il y avait un fossé. Ce fossé, cependant, Demba le franchit en même temps que le seuil de sa demeure » $(\mathrm{BD}: 22)$. En redonnant son sens propre concret à l'expression française, Birago Diop ravive la langue, il lui communique le souffle de vie africaine.

D'autre part, comme tout conte traditionnel, les Contes d'Amadou Koumba sont quelquefois l'illustration d'un proverbe ("N'Gor-Niébé ", " la Lance de l'Hyène "), ou donnent implicitement une leçon de morale ("Maman-Caïman ", "Le salaire "), et enfin le plus souvent apportent une explication d'un phénomène existant ("Fari l'ânesse", "Les mamelles ", "Les mauvaises compagnies ", "Une commission "...). Mais avec Birago Diop, les proverbes et les aphorismes deviennent partie intégrante du style et ont pour but d'annoncer l'idée initiale du conte ou introduisant un nouveau développement dans le cours de l'histoire, rendant ainsi la suite des pensées explicite et vivante. Quelques exemples éclaireront cette constatation : "L'on ne connaît l'utilité des fesses que quand vient l'heure de s'asseoir » (BD : 23). Cette phrase pleine de bon sens explique à priori ce qui va provoquer le revirement de Demba, alors que : "Qui voyage avec son aîné et son cadet fait le plus agréable des voyages " $(\mathrm{BD}: 24)$ explique le changement de l'attitude de Koumba. D'autre part, cette petite introduction-aphorisme annonce le caractère du caméléon : "Vivre seul et se moquer d'autrui, se moquer d'autrui, de ses soucis comme de ses succès, c'est là, sans conteste, un sage et raisonnable parti » (BD : 59).

Une autre caractéristique de la littérature orale que Birago Diop respecte, c'est l'usage de la répétition et " it is precisely the absence of this kind of repetition in many of the written versions of the folk tales which makes them dry and lifeless "(JAH : 27). L'illustration la plus typique de ce phénomène est ce passage des "Mauvaises Compagnies II ": " 'Djâma rek !' Que le corps est en paix, en paix seulement, ' djâma rek !', le corps souffrirait-il de dix et sept maux ; que la maison est en paix, en paix seulement, ' djâma rek!" »(BD : 68).

Qui plus est, les nombreuses descriptions de Diop sont absolument visuelles. Ainsi, par exemple, lorsqu'on lit : « Les corps se courbèrent, se plièrent, les fronts touchèrent le sable blanc comme du sucre, et les génuflexions se succédèrent au rythme des versets sacrés " (BD : 29), on voit la scène. Pour se convaincre que ces descriptions ne doivent leur existence qu'à la plume de Diop, il suffit de lire "Sarzan " : " (les calebasses de bière de mil avaient circulé de main en bouches et de bouches en mains toute la nuit) " (BD : 178) ou l'introduction réaliste de "Fari l'ânesse" (BD : 13). Une autre des tactiques de Diop pour actualiser la chose décrite est l'usage, peu fréquent il est vrai, de l'allitération, comme dans cette merveilleuse description d'une association d'idées : "Les flots fendus forment, sur le fond qui fuit, des feux follets furtifs" (BD : 32).

Diop transmet la notion de temps d'une façon très spéciale, qui donne vraiment une impression de durée : "Sept jours, puis sept jours et encore sept jours avaient passé " (BD : 25), "Il était resté sept fois sept ans dans la forêt " (BD : 43), "...en eût attrapé une indigestion pendant sept fois sept lunes " (BD : 52). Il emploie le même procédé pour donner l'impression de distance : "...parcouru la moitié de la moitié du sentier " (BD : 61) ou de foule : "...ses amis, les amis de ses amis et les amis de leurs amis 》 (BD : 81). Cependant, ce trait de style semble être africain plutôt qu'une création de Diop. En effet, on trouve sous la plume de $\mathrm{K}$. Routledge : "This happened one year, and again it happened a second and yet a third year the rain failed" (SF : 278). 
En outre, sn parlant du style de Diop, il ne faut pas oublier l'humour par lequel la subtile omniprésence de l'auteur est toujours ressentie. Cet humour est perceptible tout le long du livre et en fait se manifeste dans presque tous les exemples cités. Il nécessiterait une étude particulière à lui seul tant le sujet est riche. Il est tout de même intéressant de mentionner qu'il est très souvent exprimé de façon brève et insidieuse et par là très efficace. Ceci est évident, par exemple, dans une courte phrase à tendance ironique telle que celle-ci : « La question était trop claire pour la subtilité de ces vieillards » (BD : 26). Ou encore, un des autres procédés employés est une bride de phrase mise entre virgules ou entre parenthèses. Ainsi, lorsque Diop explique que le chien ne fréquente pas la poule aussi assidûment que le coq, ajoute-t-il : "...bien sûr et pour cause... " (BD : 80) ; lorsqu'il dit dans "Vérité et mensonge " que le mari ne se voit pas sans femme, il commente : "même mauvaise ménagère" ( $\mathrm{BD}: 131$ ).

Bien que Diop écrive en français, il semble donc nous le faire oublier par son style. Fidèle à la tradition, il lui emprunte des éléments qui lui ont inspiré une partie de son imagerie animiste, l'usage des proverbes et des répétitions ; mais l'exploitation très spéciale qu'il fait de ces données premières, les descriptions visuelles et son humour permettent à Diop de créer un style très personnel, qui paradoxalement aide à transmettre l'esprit particulier qui se dégage des contes traditionnels. Ce style, en effet, fait des contes quelque chose de divertissant, de vivant et d'authentique.

"...the superimposition of a literary over an essentially oral culture could lead sometimes not to the obliteration of the former by the latter but to the recording, codifying and preservation of elements to the oral culture and thus to making it available to an everwidening literate audience (Obiechina). En effet, si au départ les Contes d'Amadou Koumba s'adressent plutôt à un public français, de par leur langue même, dans un avenir proche, ils rempliront, comme ils commencent déjà à le faire, la mission que Diop leur a confiée : sauvegarder pour les Africains eux-mêmes, qui deviennent de plus en plus occidentaux, les racines de leurs traditions qui peu à peu sont remplacées par les valeurs occidentales.

"He resuscitates the spirit and the style of the traditional conte in beautiful French, without losing all the qualities which were in the vernacular version $"(\mathrm{JAH}: 28)$. Finalement, la tâche de Birago Diop n'est pas tellement différente de celle du griot, seuls les moyens d'expression changent, mais le côté divertissant et instructif des contes n'est en rien perdu. Ainsi, l'art de Diop réside dans l'équilibre qu'il a su trouver entre la simple traduction et la création.

Sans doute, Birago Diop a-t-il écrit inconsciemment pour le " fils du monde » (Kane 1981) dont Cheikh Hamidou Kane annonce la naissance lorsqu'il explique que les passés différents vont se fusionner en un même avenir. De par son art, Birago Diop jette un pont entre deux cultures. N'est-ce pas là le but ultime de tout traducteur?

\section{BIBLIOGRAPHIE}

BASSNETT-McGUIRE, Susan (1980) : Translation Studies, London and New York, Methuen \& Co. CENDRARS, Blaise (1963) : Anthologie nègre, Paris, Denoël, p. 402. Toute citation avec BC renvoie à cette édition. 
DIOP, Birago (1967) : Contes choisis, édit. par J.A. Hutchinson, Cambridge, Cambridge University Press, p. 9. Toute citation avec $\mathrm{JAH}$ renvoie à cette édition.

DIOP, Birago (1969) : les Contes d'Amadou Koumba, 3e éd., Paris, Présence africaine, p. 11. Toute citation avec $B D$ renvoie à cette édition.

FELDMAN, Susan (ed.) (1970) : African Myths and Tales, New York, Dell Publishing Co., pp. 144-146. Toute citation avec SF renvoie à cette édition.

HUGHES, Langston (1969) : African Treasury, New York, Crown Publishers, p. 81. Toute citation avec LH renvoie à cette édition.

KANE, Cheikh Hamidou (1971) : l'Aventure ambiguë, Mulhouse, Union-Rencontre, p. 92.

OBIECHINA, E.N. : " Transition from Oral to Literary Tradition ", Présence africaine, nº 63, p. 142. 